\title{
Contaminación microbiólogica en fórmulas infantiles en polvo en dos hospitales de Honduras
}

\section{Microbiological contamination in children's powdered formulas in two Honduran hospitals}

\section{RESUMEN}

Objetivos. Determinar la existencia de contaminación por patógenos en fórmulas infantiles en polvo (FIP) procesadas en los dos hospitales públicos más grandes de Honduras y evaluar las condiciones de procesamiento de sus servicios de fórmulas infantiles (SFI). Métodos. Estudio exploratorio realizado en dos etapas: 1) Evaluación presencial de las condiciones de procesamiento de las FIP de los dos SFI; 2) Recolección y análisis de las muestras FIP para el aislamiento de Cronobacter spp. y enterobacterias. Resultados. La evaluación de los SFI mostró debilidades en diferentes aspectos como infraestructura, almacenamiento, capacitación y registros. Cincuenta muestras fueron recolectadas en total de cinco marcas originarias de seis países. El $38 \%$ se encontraban en uso durante el muestreo y $62 \%$ fueron recolectadas de latas selladas. Se comprobó la presencia de Cronobacter spp. en 4\% (2/50) del total de muestras, una proveniente de cada hospital. Se elaboró y entregó un manual de procesamiento de FIP a cada hospital participante. Conclusiones. Existe contaminación de Cronobacter spp., Klebsiella y Acinetobacter en los dos hospitales hondureños; resultado similar a los estimados en Chile (5\%) y Cuba (1,6\%). Es necesaria la implementación del manual de procesamiento FIP y el monitoreo de estos y otros microorganismos patógenos.

Palabras clave: Acinetobacter; Cronobacter; fórmulas infantiles; infantes; microbiología de alimentos.

\footnotetext{
ABSTRACT

Objectives. Determine the existence of pathogen contamination in powdered infant formulas (PIF) processed in the two largest public hospitals in Honduras and evaluate the processing conditions of their infant formula services (IFS). Methods. Exploratory study executed in two stages: 1) faceto-face evaluation of the processing conditions of the PIF of the two IFS; 2) Collection and analysis of the PIF samples for Cronobacter spp. and Enterobacteriaceae isolation. Results. The evaluation of the IFS showed weaknesses in different aspects such as infrastructure, storage, training and keeping records. In total, fifty samples were collected, representing five brands from six countries. Thirty eight percent of samples
}

Mayra Márquez ${ }^{1}$, Adriana Hernández ${ }^{1 *}$, Jorge Wilmer Echevarría ${ }^{1}$, Omar Alejandro Tejada².

1.Departamento de Agroindustria Alimentaria, Universidad Zamorano, San Antonio de Oriente, Honduras, C.A. 2.Universidad Católica de Honduras, Tegucigalpa, D.C., Honduras, C.A.

*Dirigir correspondencia a: Adriana Hernández, Profesora del Departamento de Agroindustria Alimentaria de la Universidad Zamorano, San Antonio de Oriente, Honduras, C.A. Teléfono: 504-22872062 Email: ahernandez@zamorano.edu

Este trabajo fue recibido el 30 de octubre de 2018. Aceptado con modificaciones: 14 de enero de 2019. Aceptado para ser publicado: 23 de abril de 2019 .

were collected from cans in use during sampling and $62 \%$ were collected from sealed cans. The presence of Cronobacter spp. was detected in 4\% (2/50) of the total samples, one from each hospital. A PIF processing manual was prepared and delivered to each participating hospital. Conclusions. Contamination of Cronobacter spp., Klebsiella and Acinetobacter existed in two evaluated Honduran hospitals; results similar to others in Chile (5\%) and Cuba (1.6\%). It is necessary to implement the PIF processing manual and monitor these and other pathogenic microorganisms Keywords: Acinetobacter; Cronobacter; infant formulas; infants; food microbiology. 


\section{INTRODUCCIÓN}

El Cronobacter spp. es un género de microorganismos pertenecientes a la familia Enterobacteriaceae'. Hasta 1958 fue Ilamado Enterobacter cloacae y después renombrada como Enterobacter sakazakii en $1980^{2,3}$. Después de un estudio de su material genético (ADN) en el 2007, se realizó la propuesta de un nuevo género para la taxonomía de Enterobacter sakazakii ${ }^{4}$. En el 2008 se reclasificó en el género Cronobacter spp. que incluía cinco especies y luego se anexaron dos especies más ${ }^{1,5,6}$.

Estos microorganismos son patógenos oportunistas causantes de infecciones (exceptuando Cronobacter condimenti). En su mayoría son responsables de Enfermedades Transmitidas por Alimentos (ETA) y la mayoría de los casos están asociados a Cronobacter sakazakii y Cronobacter malonaticus ${ }^{7,8}$. El género Cronobacter spp. es de tipo Gram negativo, móvil, no formadora de esporas, anaerobias facultativas, termotolerantes y ubicuas ${ }^{9,10}$. Tienen la capacidad de formar biopelículas, son resistentes a la desecación y al estrés osmótico, y los ambientes secos favorecen su existencia. Se desconoce el reservorio pero se ha logrado aislar en diversos ambientes donde se produce leche en polvo ${ }^{11}$.

Los casos más graves y severos por infección de Cronobacter spp. están asociados al consumo de Fórmulas Infantiles en Polvo (FIP) ya que éstas no son esterilizadas y pueden estar contaminadas. Los primeros casos de infección se reportaron en el Reino Unido en el año de $1958^{12}$. En la actualidad se han presentado casos en todo el mundo causando morbilidad y mortalidad infantil principalmente $\mathrm{e}^{10,13}$.

La infección por estos microrganismos puede causar secuelas graves, solo el Cronobacter spp. es responsable de producir enfermedades como sepsis, meningitis, encefalitis, absceso cerebral y enterocolitis necrotizante e incluso hasta la muerte ${ }^{10,14}$.

Entre los grupos más afectados se encuentran los recién nacidos, los prematuros, los menores de dos meses, y los inmunológicamente deprimidos, especialmente los hijos de madres con virus de la inmunodeficiencia humana (VIH) positivo $^{9,15}$. Estos grupos tienen mayor riesgo al consumir FIP contaminadas con Cronobacter spp. porque el estómago de los recién nacidos y en especial de los prematuros es menos ácido ${ }^{16}$ en comparación con una persona adulta, lo que contribuye a la sobrevivencia del Cronobacter spp. en esta población.

La infección no suele ser frecuente, pero la mortalidad que produce es alta, entre el 40 y $80 \%{ }^{17-18}$. La dosis infectiva no se ha estimado, pero podría ser una dosis baja semejante a Escherichia coli de 10 - 100 UFC (Unidades Formadoras de Colonias). El Cronobacter spp. se encuentra con mayor frecuencia en las plantas procesadoras en comparación con la Salmonella ${ }^{10}$.

El Cronobacter spp. había sido considerado de peligro severo por la Comisión Internacional de Especificaciones Microbiológicas para los Alimentos (CIEM) y desde el 2004 la Organización de las Naciones Unidas para la Alimentación y Agricultura (FAO) conjuntamente con la Organización Mundial de la Salud (OMS) la han considerado como un microorganismo de tipo A, que lo define de peligro grave para la salud ${ }^{9}$. Según las estadísticas, existen casos de infección y muertes documentadas asociadas al consumo FIP entre 1961 y el 2008, por lo que es necesario determinar la presencia de este microorganismo, particularmente en países donde no existen registros de este microorganismo, como es el caso de Honduras.

El objetivo del presente estudio fue comprobar la contaminación de las FIP determinando la presencia de Cronobacter spp. en las fórmulas para lactantes en los dos hospitales públicos de Honduras de alcance nacional, además de evaluar las condiciones de procesamiento de sus servicios de fórmulas infantiles.

\section{MATERIALES Y MÉTODOS}

El estudio se desarrolló en dos etapas, en la primera se evaluaron presencialmente las condiciones de procesamiento de las FIP del servicio de fórmulas infantiles (SFI) de los hospitales participantes, y en la segunda etapa, se recolectaron y se analizaron las muestras.

Servicios hospitalarios y muestreo: participaron dos hospitales públicos, uno de referencia nacional (nivel complejidad 7, ubicado en la capital de Honduras) y el otro de especialidad y subespecialidades (nivel complejidad 6 , ubicado en la zona norte de Honduras). Para efectos del estudio fueron denominados como Hospital I (HI) y Hospital II (HII), considerados como los hospitales con mayor demanda de atención y mayor cantidad de partos y atenciones médicas en el país.

El tamaño de muestra se determinó considerando una población infinita determinando en 40 el número de muestras con $85 \%$ de nivel de confianza y $5 \%$ de precisión.

Para su recolección se utilizó un método aséptico preparado por los autores de acuerdo al flujo de proceso mostrado en la figura 1, que consistió en la toma de datos de cada FIP (cerrada o abierta), pesar $30 \mathrm{~g}$ y colocar la muestra en una bolsa hermética identificada con un código y a su vez, en otra bolsa estéril.

En cada muestra se recolectó información que incluyó el nombre del producto, dirección del fabricante, fecha de producción, fecha de caducidad, lote, marca, contenido neto, registro sanitario y si el frasco se encontraba en uso o sellado al momento de recoger la muestra. Las muestras se trasladaron inmediatamente en un termo cerrado al Laboratorio de Microbiología de Alimentos de la Universidad Zamorano (Municipio de San Antonio de Oriente. Francisco Morazán, Honduras).

Evaluación de los SFI: se adaptó la guía "Sistema de análisis de peligros y puntos de control crítico"19 para evaluar la elaboración de FIP en medio hospitalario. La evaluación consistió en verificar la aprobación de los aspectos considerados aceptables en cada etapa (diez etapas). De las respuestas obtenidas, se estimó un puntaje sobre el total de puntos en cada etapa evaluada (Tabla 1). 


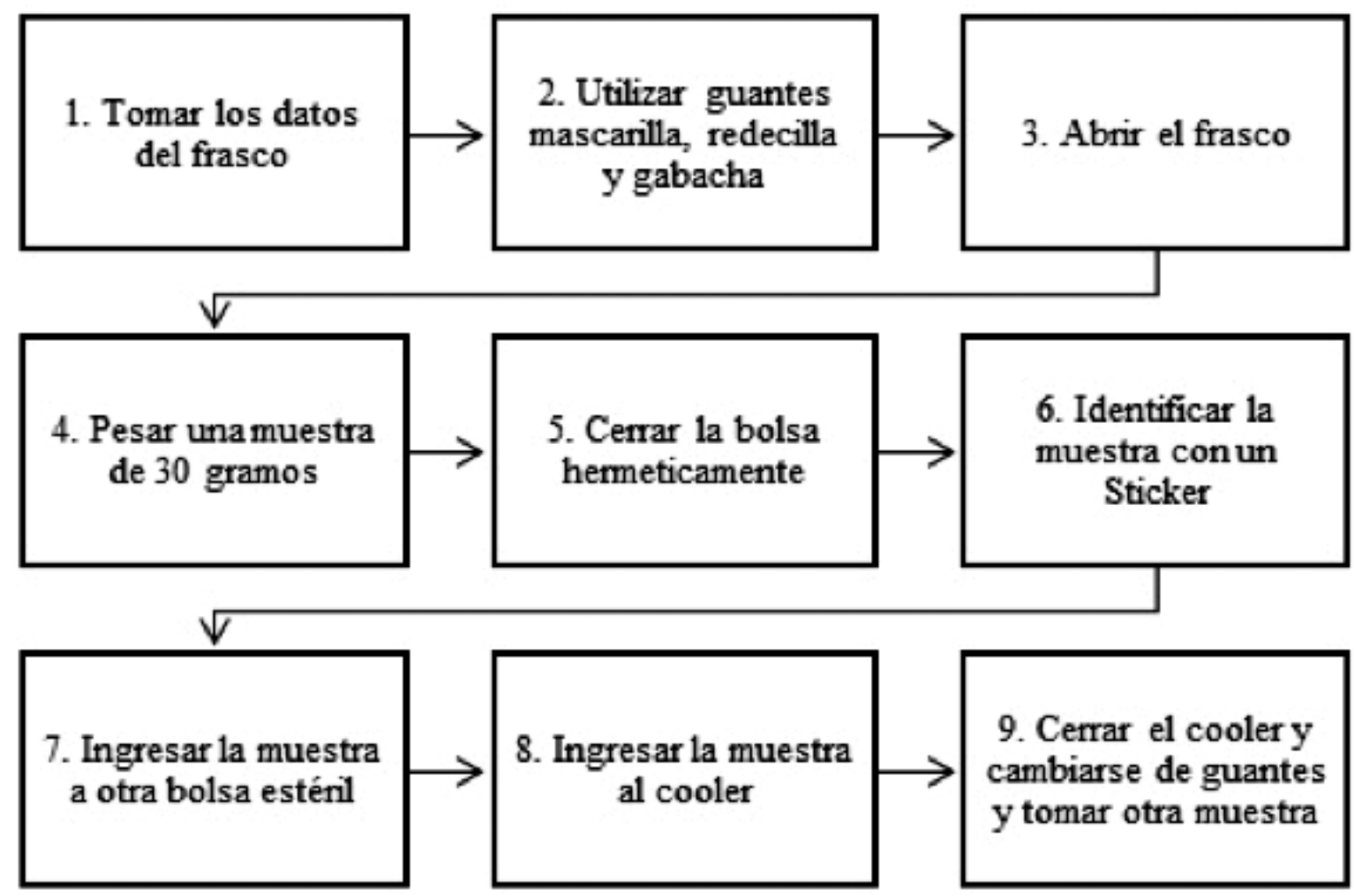

Figura 1: Flujo de proceso del método aséptico utilizado para recoger muestras de FIP, adaptado de Vargas et al (2008) ${ }^{19}$.

Tabla 1. Resultados de la evaluación del Servicio de Fórmulas Infantiles de los Hospitales I y II.

\begin{tabular}{|llcccc|}
\hline Etapa & Evaluación & Resultado *HI & \% & Resultado *HII & \% \\
\hline Inicio & Organización general del servicio FIP & $1 / 11$ & 9 & $7 / 11$ & 64 \\
$1-2$ & $\begin{array}{l}\text { Almacenajes de fórmulas infantiles y agua } \\
\text { para la reconstitución }\end{array}$ & $5 / 38$ & 13 & $14 / 38$ & 40 \\
$3-4$ & $\begin{array}{l}\text { Limpieza, desinfección y esterilización de } \\
\text { materiales y equipo de servicio }\end{array}$ & $3 / 15$ & 20 & $6 / 15$ & 45 \\
5 & $\begin{array}{l}\text { Proceso de reconstitución de las FIP } \\
6-8\end{array}$ & $\begin{array}{l}\text { Etiquetado, rellenado y transporte de } \\
\text { las fórmulas reconstituidas }\end{array}$ & $1 / 11$ & 9 & $5 / 11$ \\
Final & $\begin{array}{l}\text { Evaluación de la vigilancia del } \\
\text { procesamiento de las fórmulas }\end{array}$ & $6 / 8$ & 75 & $7 / 8$ & 88 \\
TOTAL & & $2 / 8$ & 25 & $5 / 8$ & 63 \\
\hline
\end{tabular}

*El número es la puntuación obtenida sobre el total de puntos de la etapa evaluada. Cada unidad significa la aprobación de un ítem de evaluación. 
Con esta guía se usó conjuntamente un flujo de proceso (Figura 2) para identificar los aspectos relevantes como ser: en la etapa de inicio, la organización general del servicio; en las etapas uno y dos, lo referente al almacenaje de FIP y agua para reconstitución; en las etapas tres y cuatro, la limpieza, desinfección y esterilización de los materiales y equipos; en la etapa cinco, el proceso de reconstitución de las FIP; en las etapas de la seis a la ocho, lo referente al etiquetado, rellenado y transporte de las fórmulas reconstituidas; en las etapas nueve y diez, la refrigeración y almacenamiento de las fórmulas reconstituidas; en la once, la preparación de los contenedores para el consumo, y en la etapa final, la evaluación de la vigilancia. Debido a que en ambos hospitales las FIP reconstituidas son transportadas inmediatamente para el consumo, las etapas 9-10 no fueron evaluadas.

Análisis microbiológico: para la identificación de Cronobacter spp. se utilizó el método de especificaciones técnicas - ISO/TS 22964:2006 ${ }^{13,20}$. La cantidad de muestra utilizada fue de 10 gramos, tomando en cuenta los criterios microbiológicos aplicables a los productos alimenticios de la Comisión Europea para el método ISO/TS 22964:2006 ${ }^{21}$. El aislamiento selectivo se realizó con CHROMagar ${ }^{\mathrm{TM}}$ para Cronobacter spp. y para la confirmación bioquímica

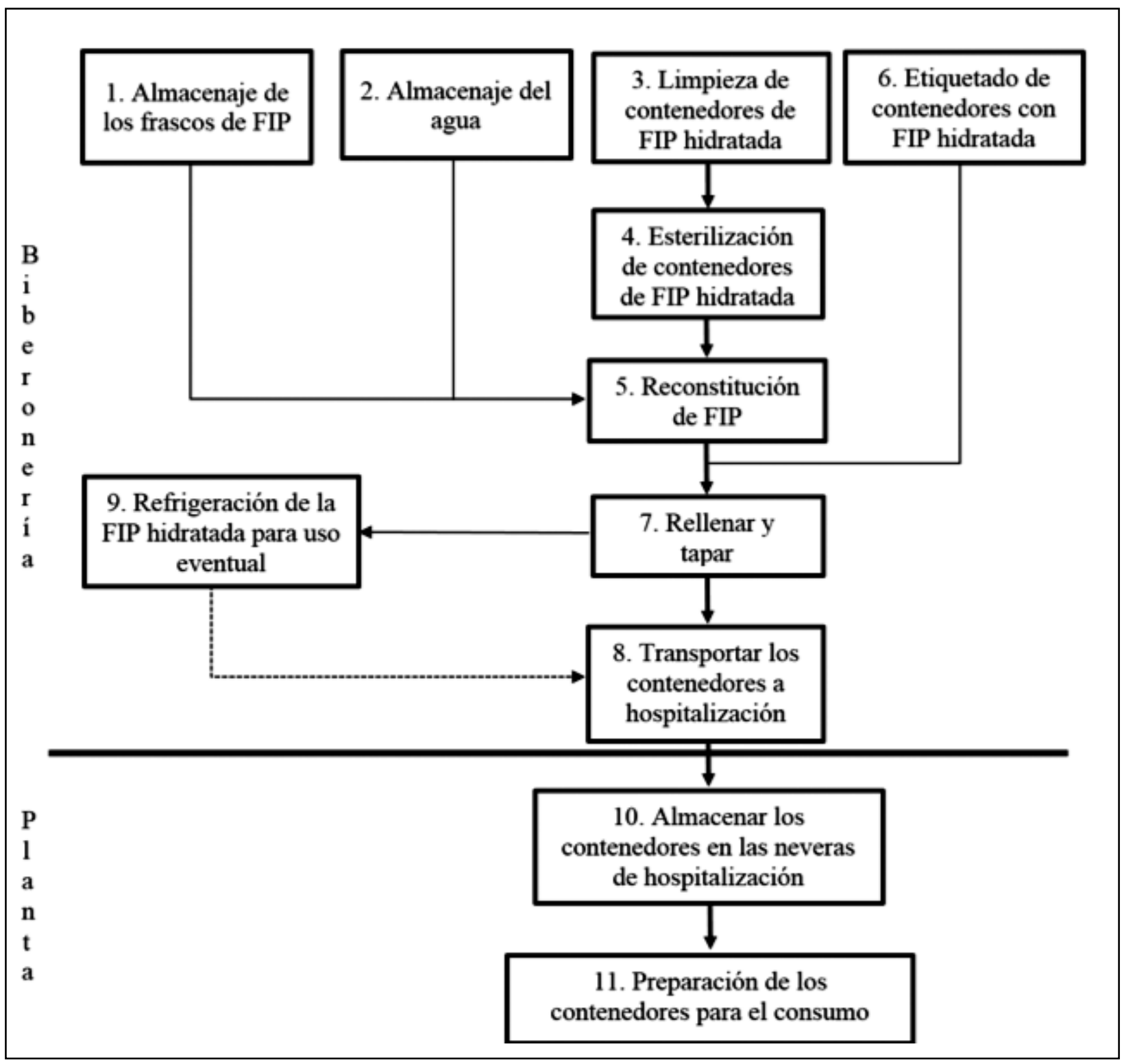

Figura 2: Flujo de proceso de procesamiento de FIP en hospitales, adaptado de Vargas et al (2008) ${ }^{19}$. 
MicrogenTM GN-ID Identificación. El procedimiento se realizó según el flujo del proceso de la figura 3.

Análisis estadístico: se utilizaron frecuencias absolutas y porcentajes para las variables cualitativas.

\section{RESULTADOS}

La evaluación de los SFI en los dos hospitales con la guía adaptada "Sistema de análisis de peligros y puntos de control crítico $^{\prime 19}$, tuvo como resultado la identificación de diferentes falencias durante el proceso de reconstitución de FIP para obtener un producto inocuo (Tabla 1).

En el hospital I y de acuerdo a la guía, la infraestructura no aseguraba un proceso que garantizara la inocuidad de las FIP al ser reconstituidas ni tampoco contaban con las condiciones correctas para su almacenamiento. No se observó un control sobre las temperaturas dentro del área de procesamiento para poder reducir la posibilidad de crecimiento de microorganismos y en especial de Cronobacter spp. El nivel educativo del personal que procesa las FIP en este hospital no es el adecuado; no se encontraba capacitado y desconocía los peligros de procesar inadecuadamente las FIP.

En el hospital II, la evaluación identificó mejores condiciones de procesamiento de las FIP, en comparación al hospital I. Aun así, los procedimientos no garantizaban que las fórmulas rehidratadas fueran inocuas; esto debido al escaso conocimiento para reconstituir fórmulas y a que el personal no reconocía los peligros durante su procesamiento. No se encontraron flujos de procesos ni registros para la reconstitución de las FIP.

En las visitas a ambos hospitales se recolectaron un total de 50 muestras de FIP que provenían de 14 productos, de cinco marcas disponibles en el mercado, originarias de seis países (Honduras no es productor de FIP) que se encontraban disponibles en el momento de la recolección. El 38\% (19/50) de las fórmulas procesadas se encontraban en uso durante el muestreo y el $62 \%(31 / 50)$ de muestras restantes fueron recolectadas de latas selladas.

Se identificó Cronobacter spp. con una frecuencia del 4\% (2/50) en FIP procesadas en los dos hospitales, una muestra de cada hospital participante en el estudio. Las especies que se encontraron corresponden a Cronobacter sakazakii.

\section{DISCUSIÓN}

Está demostrado que la contaminación y deficiencias en la aplicación de prácticas de higiene durante la producción

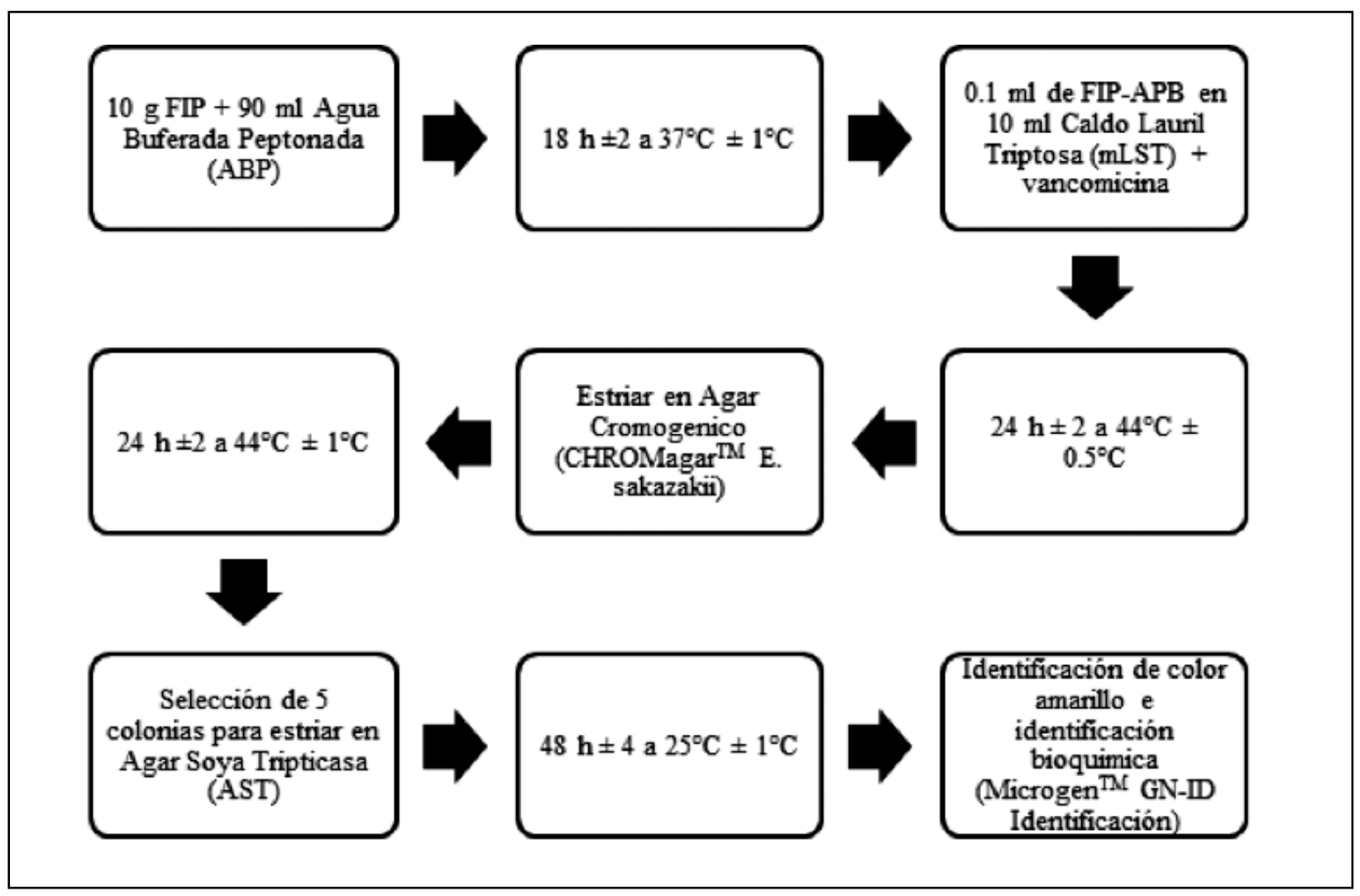

Figura 3: Flujo de proceso del Método ISO/TS 22964:200620 para aislamiento de Cronobacter sakazakii en FIP. 
de las FIP, no son los únicos factores determinantes para que exista Cronobacter spp., porque su presencia se ha determinado hasta en FIP que cumplían con los requisitos de la Comisión del Codex Alimentarius (FAO/OMS) para el recuento de coliformes $(<3 \mathrm{UFC} / \mathrm{g})^{16}$. Las deficientes prácticas de higiene al momento de la reconstitución en los hospitales u hogares y la exposición a temperaturas de no refrigeración $\left(>5^{\circ} \mathrm{C}\right)$, aumentan la posibilidad de la infección en el momento del consumo de la FIP. No se descarta que el equipo que se usa en la preparación de FIP se haya visto asociado a contaminación, causando brotes ${ }^{10}$.

Los resultados del presente estudio corresponden al primer reporte científico sobre la contaminación de FIP con Cronobacter spp. en hospitales de Honduras. Este resultado es similar al encontrado en el primer aislamiento de Cronobacter spp. en Chile (2012) en una planta procesadora de FIP en el que se utilizó el mismo protocolo ISO/TS 22964:2006 y se reportó el $5 \%$ de muestras confirmadas de 80 procesadas $^{17}$. Mas recientemente (2015), otro estudio en Chile estimó $2.7 \%$ de prevalencia de este microrganismo en 72 muestras $^{22}$. En las conclusiones de ambos estudios se solicitaba que el Cronobacter spp. sea considerado como un riesgo por el Reglamento Sanitario de los Alimentos del mencionado país, porque debe estar ausente en las FIP, y realizar su búsqueda clínica a través de análisis microbiológico sistemático de las preparaciones lácteas ${ }^{23}$.

En Cuba (2008), se reportó la presencia de Cronobacter spp. en 1,6\% de las muestras analizadas de un total de 60 muestras procesadas. La importación de las FIP provenía de nueve países. Para la identificación se usó el método del FDA (Food and Drug Administration) ${ }^{24}$. En Colombia (2009), también se ha reportado la presencia de Cronobacter spp. con el $27,7 \%$, de muestras positivas, de 222 muestras procesadas de nueve lactarios, analizadas con el método $\mathrm{FDA}^{25}$.

No se puede asociar a una misma fuente de contaminación de Cronobacter spp. a todas las FIP, por pertenecer a diferentes marcas, lotes y países. Para ello, sería necesario realizar un análisis de huella genética para poder reportar el perfil y para rastrear posibles fuentes de contaminación.

Por estudios realizados se desconoce el reservorio de Cronobacter spp., pero se ha logrado aislar en diversos ambientes donde se produce leche en polvo, además en plantas donde se procesan otros alimentos y en hogares ${ }^{11}$. Castro et al., refieren que la contaminación con Cronobacter spp. se puede llevar a cabo en la fabricación o en el momento de la reconstitución de las fórmulas ${ }^{18}$, debido a que esta bacteria puede encontrarse en el medio ambiente y en los alimentos ${ }^{26,27}$. También se transfiere a las FIP por malas prácticas de manufactura en la fabricación, por agregar ingredientes contaminados después de la pasteurización, por contaminación directa en el equipo, y por personas que manipulan el alimento y son portadoras ${ }^{28}$.

El tiempo de reproducción bacteriana para doblar su población es de 300 minutos en $10^{\circ} \mathrm{C}$, que se reduce a 40 minutos a una temperatura de $23^{\circ} \mathrm{C}$ y a 20 minutos a $37^{\circ} \mathrm{C}$, por lo que se puede multiplicar durante el almacenamiento y procesamiento ${ }^{28}$.

El riesgo de contaminación aumenta debido a que este microorganismo tiene resistencia al calor, considerándose como el microorganismo más termoresistente de las enterobacterias ${ }^{9,26}$ y está relacionado con casos de meningitis, septicemia, enterocolitis necrotizante y sepsis en recién nacidos por el consumo de FIP contaminadas ${ }^{29}$, como se determinó en pacientes hospitalizados en México (2011) con diarrea hemorrágica por Cronobacter spp.

México dispone de su Norma Oficial NOM-131SSA1-2012 enfocada a fórmulas para lactantes de observancia obligatoria para las personas físicas y morales que se dedican al proceso y/o importación de los productos objeto de la misma, que van a ser comercializados en el territorio nacional ${ }^{30}$.

En los dos hospitales evaluados en este estudio, como ocurrió en Chile, Cuba y Colombia, existe exposición a Cronobacter spp. cuyas condiciones promueven la rápida proliferación del microorganismo que puede sobrevivir hasta dos años con una actividad de agua de 0,14-0,27 que le permite estar presente en alimentos deshidratados ${ }^{10,27}$.

Además, se encontró presencia de Klebsiella spp. en $8 \%$ de las muestras, asociada como causante de una gran variedad de patologías, incluyendo infecciones gastrointestinales ${ }^{31}$; Acinetobacter en 4\%, asociada con altos porcentajes de mortalidad, con alta capacidad de diseminarse en el ambiente hospitalario ${ }^{32}$, de importancia intrahospitalaria mundial por el alto nivel de resistencia a

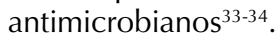

La Klebsiella spp. representa un peligro en las FIP ya que podría estar presente en las fórmulas importadas ${ }^{17,35}$. También se identificó Yersinia spp., cuya contaminación es causa común de enfermedades de transmisión alimentaria.

Se elaboró y entregó a los dos hospitales participantes, un manual de reconstitución de FIP, previamente revisado por especialistas del Departamento de Agroindustria Alimentaria de la Universidad Zamorano, que considera los procedimientos requeridos por la FAO y las OMS para procesar fórmulas infantiles 36 , en los que se incluyeron capítulos sobre establecimiento de registros y estandarización de procedimientos, almacenamiento de FIP, limpieza, desinfección y esterilización de material y equipo y los procedimientos para la reconstitución de las FIP.

La implementación del manual es importante porque existe peligro potencial de contaminación en las FIP, debido al carente conocimiento sobre el procesamiento de las fórmulas y las temperaturas de manejo que se usan en ambos hospitales para su reconstitución que contribuyen al crecimiento poblacional de Cronobacter spp.

De ahí que se requieren implementar mejoras en la organización, registros, capacitación y seguimiento para procesar FIP, basados en las recomendaciones de la OMS/ FAO y con ello evitar contaminación e infección en pacientes. 
Se recomienda realizar monitoreo e investigación por parte de las instancias responsables en alianza con organizaciones privadas y académicas, y extender el estudio a todos los hospitales del país y la región centroamericana, dada la similitud de las condiciones. Además, promover la lactancia materna exclusiva como la alimentación óptima para el lactante, sobre todo al recién nacido y en condiciones de prematurez.

\section{Limitaciones y fortalezas}

No se pudo determinar si ambas muestras positivas provenían contaminadas de fábrica o la contaminación del producto tuvo lugar en la reconstitución de las fórmulas en los hospitales, debido a que ambas fórmulas contaminadas pertenecían a productos que se encontraban en uso en el momento del muestreo. Los resultados del estudio se limitan a los dos hospitales más grandes y complejos de Honduras, siendo el primero en este tipo.

Agradecimiento. El estudio fue financiado por la OPS/ OMS por lo que los autores agradecen el apoyo recibido para su realización.

Conflicto de intereses. Los autores declaran que no existieron conflictos de ningún tipo en la realización de esta investigación.

\section{BIBLIOGRAFIA}

1. Iversen C, Lehner A, Mullane N, Bidlas E, Cleenwerck I, Marugg J, Fanning S, Stephan R, Joostenet $H$. The taxonomy of Enterobacter sakazakii: proposal of a new genus Cronobacter gen. nov. and descriptions of Cronobacter sakazakii comb. nov. Cronobacter sakazakii subsp. sakazakii, comb. nov., Cronobacter sakazakii subsp. malonaticus subsp. nov., Cronobacter turicensis sp. nov., Cronobacter muytjensii sp. nov., Cronobacter dublinensis sp. nov. and Cronobacter genomospecies 1. BMC Evol Biol 2007; 7: 64-74. DOI: 10.1186/1471-2148-7-64

2. Farmer I, Asbury M, Hickman F, Brenner D. Enterobacter sakazakii: A New Species of "Enterobacteriaceae" Isolated from Clinical Specimens. Inter J of Syst Bacteriol 1980; 30:569-584. DOI: 10.1099/00207713-30-3-569

3. Chen Y, Lampel K, Hammack T. BAM: Cronobacter (chapter 29). Bacteriological Analytical Manual. U.S. Food and Drug Administration: 2012. https://www.fda.gov/food/ foodscienceresearch/laboratorymethods/ucm289378. htm. Access on August 14, 2016.

4. Iversen C. Mullane N, McCardell B, Tall B, Lehner A, Fanning $S$, Stephan $R$, Joosten $H$. Cronobacter gen. nov., a new genus to accommodate the biogroups of Enterobacter sakazakii, and proposal of Cronobacter sakazakii gen. nov., comb. nov., Cronobacter malonaticus sp. nov., Cronobacter turicensis sp. nov., Cronobacter muytjensii sp. nov., Cronobacter dublinensis sp. nov., Cronobacter genomospecies 1, and of three subspecies, Cronobacter dublinensis subsp. dublinensis subsp. nov., Cronobacter dublinensis subsp. lausannensis subsp. nov. and Cronobacter dublinensis subsp. lactaridi subsp. nov. Int J Syst Evol Microbiol 2008; 58: 1442-1447.
DOI: 10.1099/ijs.0.65577-0

5. Healy B, Cooney $S, O^{\prime}$ Brien $S$, Iversen $C$, Whyte $P$, Nally I, Callanan J, Fanning S. Cronobacter (Enterobacter sakazakii): an opportunistic foodborne pathogen. Foodborne Pathog Dis. 2010; 7(4): 339-350. DOI: 10.1089/fpd.2009.0379

6. Joseph S, Cetinkaya E, Drahovska H, Levican A, Figueras MJ, Forsythe SJ. Cronobacter condimenti sp. nov., isolated from spiced meat, and Cronobacter universalis sp. nov., a species designation for Cronobacter sp. genomospecies 1, recovered from a leg infection, water and food ingredients. Int J Syst Evol Microbiol 2012; 62: 1277-1283. DOI:10.1099/ ijs.0.032292-0

7. Sigüenza T. Outbreaks caused by Cronobacter sakazakii. University of Valladolid. Spain, 2014. http://uvadoc.uva.es/ handle/10324/5623

8. Asato V, Vilches VE, Pineda MG, Casanueva E, Cane A, Moroni MP et al. First clinical isolates of Cronobacter spp. (Enterobacter sakazakii) in Argentina: Characterization and subtyping by pulsed-field gel electrophoresis. Rev Arg Microbiol 2013; 45(3): 163-164. https://www.ncbi.nlm.nih. gov/pubmed/24165138.

9. WHO/FAO. Enterobacter sakazakii and other microorganisms in powdered infant formula. Meeting report. Microbiological risk assessment series. OMS/FAO. Rome. 2004. http://apps. who.int/iris/handle/10665/43682.

10. WHO/FAO. Enterobacter sakazakii (Cronobacter spp.) in powdered follow-up formula. Meeting report. Microbiological Risk Assessment Series 15. Nutrition and Consumer Protection Division. FAO. Department of Food Safety, Zoonoses and Foodborne Disease. WHO. 2008. http://www.who.int/ foodsafety/publications/mra_followup/en/.

11. Agencia Catalana de Seguridad Alimentaria. Cronobacter spp. Catalan Agency for Food Safety. SSN: 2013-3308. Legal Dep: B. 24469-2012. 2013. http://acsa.gencat.cat/ca/inici. Access on September 19, 2016.

12. Urmenyi A, Flanklin A. Neonatal death from pigmented coliform infection. Lancet 1961; 277: 313-315. DOI: 10.1016/ S0140-6736(61)91481-7

13. Leotta G, Pacheco S, Epszteyn S, Lirón JP, Stambullian J, Vecchiarelli C, Stamboulian D. Cronobacter sakazakii in Infantile Milk Formulas. Foundation Center for Infectious Studies. Argentina. 2011. 288p. Available on: http://www. funcei.org.ar/upload/nota/documentos/publicaciones11_1. $p d f$

14. Cortés-Sánchez A, Espinosa-Chaurand LD. Cronobacter sakasakii a potential health hazard from food. Rev de aplicación científica y técnica. 2018; 4(11): 7-19 http://www.ecorfan. org/spain/researchjournals/Aplicacion_Cientifica_y_Tecnical vol4num11/Revista_de_Aplicacion_Cientifica_y_Tecnica_ V4_N11_2.pdf

15. Codex Alimentarius. Code of Hygienic Practice for powdered preparations for infants and young children. CAC/RCP $66-$ 2008. www.fao.org/fao-who-codexalimentarius/codex-texts/ codes-of-practice/es/. Access on September 28, 2016.

16. Report of the Scientific Committee of the Spanish Agency for Consumer Affairs, Food Safety and Nutrition (AECOSAN) on the microbiological risks associated with the consumption of certain foods by children from 0 to 3 years old. 2015. AECOSAN-2015-006. Scientific committee journal \#22. http:// www.aecosan.msssi.gob.es/AECOSAN/docs/documentos/ seguridad_alimentaria/evaluacion_riesgos/informes_comite/ MICRO_NINOS_O_3_ANIOS.pdf 
17. Sáez M, Llanos S, Tamayo R. First isolation of Cronobacter spp (Enterobacter sakazakii) in powdered milk formula produced in Chile. Rev Chi Salud Pub. 2012; 16(1): 11-15. https://revistas.uchile.cl/index.php/RCSP/article/view/18607.

18. Castro J, Gómez C, Vargas E. Cronobacter sakazakii. Chemical Research Center. Institute of Basic Sciences of Engineering, Universidad Autónoma del Estado de Hidalgo, México. 2013. 45p. http://www.ciq.uaem.mx/.

19. Vargas-Leguás H et al. Guide for the preparation of powdered infant formula in hospitals. Hazard analysis system and critical control points. An Pediatr. 2009; 70(6); 586-593. https://doi.org/10.1016/j.anpedi.2009.01.006

20. International Standards for Organization. 2006. Milk and milk products-Detection of Enterobacter sakazakii. ISO/ TS 22964:2006 (IDF/RM 210: 2006). https://www.iso.org/ standard/41258.html. Access on August 23, 2016.

21. European Commission. Regulation (CE) no. 2073/2005 of the commission of November 15, 2005 relating to the microbiological criteria applicable to food products. European Commission. 2007. 33p. https://eur-lex.europa.eu/ legal-content/ES/TXT/?uri=celex\%3A32005R2073. Access on September 23, 2016.

22. Parra J, Oliveras L, Rodríguez A, Riffo F, Jackson E, Forsythe S. Risk of Cronobacter sakazakii contamination in powdered milk for infant nutrition. Rev Chil Nutr 2015; 42(1): 83-9. https:// scielo.conicyt.cl/scielo.php?script=sci_abstract\&pid=S071775182015000100011\&lng=es\&nrm=iso\&tlng=es

23. Bustos AE, Franulic PY, Farías ZN. Estándares de calidad para un servicio dietético de leche, en un hospital de niños con enfermedades crónicas. Rev. chil. nutr. 2016; 43(1): 92-97. https://scielo.conicyt.cl/scielo.php?script=sci_ arttext\&pid=S0717-75182016000100014\&/ng=es. http:// dx.doi.org/10.4067/S0717-75182016000100014.

24. Leyva V, Ruiz H, Machín M, Tejedor R, Martino T, Ferrer $Y$. First study of Enterobacter sakazakii in foods in Cuba. Rev Cub Salud Púb. 2008; 34. http://scielo.sld.cu/scielo.php?script=sci_ arttext\&pid=S0864-34662008000400008\&Ing=es

25. Vanegas $M$, Rugeles $L$, Martínez A. Isolation and identification of Enterobacter sakazakii in milk feeders of Bogotá, D. C. Asociación colombiana de infectología. Revista infectio. 2009; 13:36-42. http://www.scielo.org.co/scielo.php?script=sci_ar ttext\&pid=S0123-93922009000100005.

26. Petrola M. Evaluation of some factors that affect the development of Cronobacter sakazakii in Infantile Milk Formulas. Universidad Central de Venezuela. Caracas, Venezuela. 2012. 59p. http:// saber.ucv.ve/bitstream/123456789/3335/1/T026800002765O-trabajodegrado6 Maribel.Petrola-000.pdf

27. Carbajal D, Paredes M. Study of the presence of Enterobacter sakazakii in children's powdered formulas provided to nursing patients of a public hospital in the city of Guayaquil. Escuela Superior Politécnica del Litoral. 2013. 53p. https:// www.dspace.espol.edu.ec/retrieve/89705/D-79795.pdf

28. Elika. Cronobacter. Basque Foundation for Food and Agriculture Security. 2013. 3p. https://www.elika.eus/ infogramas-riesgos/\#1516697303693-3ae81f88-f6ac. Access on September 23, 2016.

29. Flores J, Arvizu S, Silva J, Fernández E. Two cases of hemorrhagic diarrhea caused by Cronobacter sakazakii in hospitalized nursing infants associated with the consumption of powdered infant formula. J Food Prot 2011; 74(12): 2177-2181. DOI: 10.4315/0362-028X.JFP-11-257.

30. Norma Oficial Mexicana NOM-131-SSA1-2012. Productos y servicios. Fórmulas para lactantes, de continuación y para necesidades especiales de nutrición. Alimentos y bebidas no alcohólicas para lactantes y niños de corta edad. Disposiciones y especificaciones sanitarias y nutrimentales. Etiquetado y métodos de prueba. Diario oficial. México. 2012.

31. Albarado LS, Flores EM, Mendoza GMT. Association of Klebsiella spp., with acute diarrheal syndrome in children 0 to 2 years of age. Salus Rev Fac Ciencias Salud. University of Carabobo [on line] 2007; 11(3): 7-12. http://udg.redalyc. org/articulo.oa? id=375938985003. ISSN 1316-7138. Access on October 26, 2018.

32. Vanegas-Múnera JM, Roncancio-Villamil G, Jiménez-Quiceno JN. Acinetobacter baumannii: clinical importance, resistance mechanisms and diagnosis. Rev CES Med 2014; 28(2): 233246. http://www.scielo.org.co/pdf/cesm/v28n2/v28n2a08. pdf.

33. Santisteban Y, Carmina Y, Pérez Y, Díaz L, García S, Kobayashi N, Quiñonez D. Infections by the Klebsiella and Acinetobacter genera in Cuban pediatric hospitals and antibiotic resistance. Habana. Rev Cub Med Trop 2014; 66 (3): 400-414. http://scielo.sld.cu/scielo.php?script=sci_artt ext\&pid=S0375-07602014000300008

34. Agodi A, Barchitta M, Quattrocchi A, Maugeri A, Aldisio E, Marchese A, Mattaliano A, Tsakris A. Antibiotic trends of Klebsiella pneumoniae and Acinetobacter buamannii resistance indicators in an intensive care unit of Southern Italy, 2008-2013. Antimicrob Resist Infect Control. 2015; 4: 43. DOI: 10.1186/s13756-015-0087-y

35. Parra J. Microbial Risk Assessment in foods prepared in a Maternal and Child Hospital. Universidad Autónoma de Querétaro. Querétaro, México. 2011. 192p. http://ri.uaq. mx/bitstream/123456789/906/1/RI000075.pdf

36. WHO/FAO. Preparation, storage and handling under hygienic conditions of powder preparations for infants. Guidelines. 2007. 30p. http://www.who.int/publications/ list/9789241595414/es/. Access on August 16, 2016. 